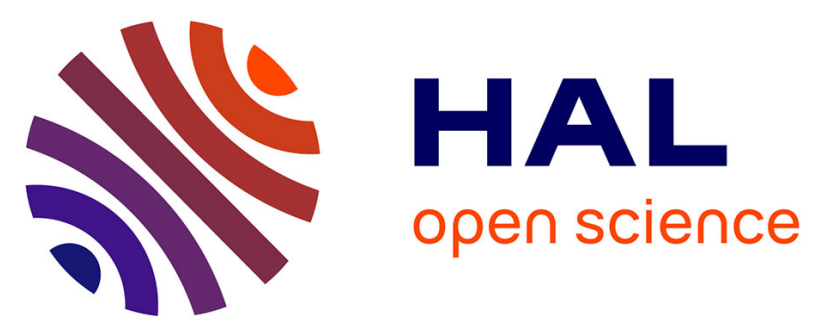

\title{
Simultaneous Measurement of Metabolite Concentration and Isotope Incorporation by Mass Spectrometry
}

Maud Heuillet, Pierre Millard, Madi y Cissé, Laetitia K Linares, Fabien

Letisse, Serge Manié, Laurent Le Cam, Jean-Charles Portais, Floriant Bellvert

\section{- To cite this version:}

Maud Heuillet, Pierre Millard, Madi y Cissé, Laetitia K Linares, Fabien Letisse, et al.. Simultaneous Measurement of Metabolite Concentration and Isotope Incorporation by Mass Spectrometry. Analytical Biochemistry, 2020, 92 (8), pp.5890-5896. 10.1021/acs.analchem.9b05709 . hal-02563842

HAL Id: hal-02563842

https://hal-univ-rennes1.archives-ouvertes.fr/hal-02563842

Submitted on 14 May 2020

HAL is a multi-disciplinary open access archive for the deposit and dissemination of scientific research documents, whether they are published or not. The documents may come from teaching and research institutions in France or abroad, or from public or private research centers.
L'archive ouverte pluridisciplinaire HAL, est destinée au dépôt et à la diffusion de documents scientifiques de niveau recherche, publiés ou non, émanant des établissements d'enseignement et de recherche français ou étrangers, des laboratoires publics ou privés. 


\title{
Simultaneous measurement of metabolite concentration and isotope incorporation by mass spectrometry
}

\author{
Maud Heuillet ${ }^{1,2, \ddagger}$, Pierre Millard ${ }^{1, \ddagger}$, Madi Y. Cissé ${ }^{3}$, Laetitia K. Linares ${ }^{3}$, Fabien Létisse ${ }^{1}$, Serge Manié ${ }^{4}$, \\ Laurent Le Cam ${ }^{3}$, Jean-Charles Portais ${ }^{1,2,5}$ and Floriant Bellvert ${ }^{1,2, *}$ \\ ${ }^{1}$ TBI, Université de Toulouse, CNRS, INRAE, INSA, UPS, Toulouse, 31077, France \\ ${ }^{2}$ MetaboHUB-MetaToul, National Infrastructure of Metabolomics and Fluxomics, Toulouse, 31077, France \\ ${ }^{3}$ IRCM, Institut de Recherche en Cancérologie de Montpellier, INSERM U1194, Université de Montpellier, Institut régional du Cancer de \\ Montpellier, Montpellier, F-34298, France \\ ${ }^{4}$ INSERM U1242, Université de Rennes, Rennes, France \\ ${ }^{5}$ STROMALab, Université de Toulouse, INSERM U1031, EFS, INP-ENVT, UPS, Toulouse, France
}

\begin{abstract}
Studies of the topology, functioning and regulation of metabolic systems are based on two main types of information which can be measured by mass spectrometry: the (absolute or relative) concentration of metabolites and their isotope incorporation in ${ }^{13} \mathrm{C}$-labeling experiments. These data are currently obtained from two independent experiments because the ${ }^{13} \mathrm{C}$-labeled internal standard (IS) used to determine the concentration of a given metabolite overlaps the ${ }^{13} \mathrm{C}$-mass fractions from which its ${ }^{13} \mathrm{C}$ isotopologue distribution (CID) is quantified. Here, we developed a generic method with a dedicated processing workflow to obtain these two information simultaneously in a unique sample collected from a single cultivation, thereby reducing by a factor of two both the number of cultivations to perform and the number of samples to collect, prepare and analyze. The proposed approach is based on an IS labeled with other isotope(s) which can be resolved from the ${ }^{13} \mathrm{C}$-mass fractions of interest. As proof-of-principle, we analyzed amino acids using a doubly labeled ${ }^{15} \mathrm{~N}^{13} \mathrm{C}$-cell extract as IS. Extensive evaluation of the proposed approach shows a similar accuracy and precision compared to state-of-the-art approaches. We demonstrate the value of this approach by investigating the dynamic response of amino acids metabolism in mammalian cells upon activation of the PERK kinase, a key component of the unfolded protein response. Integration of metabolite concentrations and isotopic profiles reveals a reduced de novo biosynthesis of amino-acids upon PERK activation. The proposed approach is generic and can be applied to other (micro)organisms, analytical platforms, isotopic tracers, or classes of metabolites.
\end{abstract}

Our capacity to analyze the topology, functioning and regulation of metabolic systems is vital to understand and master the behavior of (micro)organisms in systems biology, biotechnology or biomedical research ${ }^{1-5}$. Mass spectrometry is a valuable tool for metabolic studies and is widely used to provide two main types of information: the concentration of metabolites and the incorporation of ${ }^{13} \mathrm{C}$ atoms into metabolites (Carbon Isotopologue Distribution, CID) in ${ }^{13} \mathrm{C}$-isotope labeling experiments $\left({ }^{13} \mathrm{C} \text {-ILEs }\right)^{6}$. Coupling metabolite quantification with isotope tracing is critical to understand the operation and dynamics of metabolic systems in vivo, e.g. to measure metabolic fluxes ${ }^{5,7-10}$ or to understand properties of metabolic systems such as flux control ${ }^{5,11,12}$. The most robust and accurate approaches for (absolute or relative) metabolite quantification requires an internal standard (IS), typically an uniformly ${ }^{13} \mathrm{C}$-labeled cellular extracts when using the state-of-the-art Isotope Dilution Mass Spectrometry (IDMS) approach ${ }^{13-16}$. Since the ${ }^{13}$ C-IS cannot be resolved from the ${ }^{13} \mathrm{C}$-mass fractions and affects the CID measurement, two cultivations must be conducted independently: one with an unlabeled substrate (for metabolite quantification) and one with a ${ }^{13} \mathrm{C}$-labeled tracer (for isotopic analyses). This conceptual limitation increases the number of cultures and samples to collect and analyze and may thus be difficult to implement to investigate dynamic metabolic states (for which samples must be taken at multiple time-points) or when the biological material is limited (e.g. stem cells). As an additional concern, metabolite concentrations and isotopic data are obtained from distinct cultivations where metabolic states might differ to some extend due to biological variability. Current experimental approaches therefore impair data integration due to biological variability, which may biased biological interpretations.

In this work, we developed a generic approach allowing simultaneous quantification of CIDs and metabolite pools based on a doubly-labeled IS. Doubly-labeled cell extracts have proved useful to identify and quantify metabolites in unlabeled samples ${ }^{17-19}$, and we show they are equally valuable to quantify the concentration and CIDs of metabolites in the context of isotope labeling experiments, where metabolites are isotopically enriched. As a proof of concept, this new method was first evaluated for the analysis of intracellular amino acids, using a ${ }^{15} \mathrm{~N}^{13} \mathrm{C}$-IS. It was then applied to investigate the regulation of amino acids metabolism by the PERK kinase in rat fibroblasts.

\section{EXPERIMENTAL SECTION}

Chemicals and Reagents. All unlabeled amino acids, formic acid (98\% LC-MS grade), acetonitrile (LC-MS grade) and methanol (LC-MS grade) were purchased from Sigma-Aldrich (St. Louis, MO, USA). U-13 C-glucose and ${ }^{15} \mathrm{NH}_{4} \mathrm{Cl}$ were obtained from Eurisotop (St. Aubin, France). Water obtained from a MilliQ-Plus ultrapure water purification system (Millipore, Bedford, MA, USA) was used to prepare all samples, extraction solutions and mobile phases. 
Preparation of the ${ }^{13} \mathrm{C}^{15} \mathrm{~N}$-internal standard. Escherichia coli $\mathrm{K}-12$ MG1655 was grown on M9 minimal medium ${ }^{20}$ containing 5 $\mathrm{mM} \mathrm{KH}_{2} \mathrm{PO}_{4}, 10 \mathrm{mM} \mathrm{Na}_{2} \mathrm{HPO}_{4}, 9 \mathrm{mM} \mathrm{NaCl}, 0.8 \mathrm{mM} \mathrm{MgSO}_{4}$, $0.1 \mathrm{mM} \mathrm{CaCl} 2,0.3 \mathrm{mM}$ of thiamine, $40 \mathrm{mM}{ }^{15} \mathrm{NH}_{4} \mathrm{Cl}$ as sole nitrogen source, and $15 \mathrm{mM}$ of either ${ }^{12} \mathrm{C}$ - or $\mathrm{U}-{ }^{13} \mathrm{C}$-glucose as sole carbon source. Glucose and thiamine were sterilized by filtration, the other compounds were autoclaved separately. LB overnight cultures $(5 \mathrm{~mL})$ were used to inoculate shake flasks containing $10 \mathrm{ml}$ of minimum medium, at an initial $\mathrm{OD}_{600 \mathrm{~nm}}$ of 0.05 , and incubated at $37{ }^{\circ} \mathrm{C}$ and $200 \mathrm{rpm}$. Cells were harvested during the exponential growth phase $\left(\mathrm{OD}_{600 \mathrm{~nm}} \approx 2\right)$ by centrifugation (10 min, $10000 \mathrm{~g}$, room temperature) with a Sigma $3-18 \mathrm{~K}$ centrifuge, washed with the same volume of fresh medium, and used to inoculate at an initial $\mathrm{OD}_{600 \mathrm{~nm}}$ of 0.05 a $1 \mathrm{~L}$ baffled flasks $\left(37^{\circ} \mathrm{C}, 200 \mathrm{rpm}\right)$ containing $200 \mathrm{~mL}$ of medium sparged with synthetic gas $80 \% \mathrm{~N}_{2} / 20 \% \mathrm{O}_{2}$ (containing less than 1 ppmv $\mathrm{CO}_{2}$, Air Liquide, France). Cell growth was monitored by measuring optical density at $600 \mathrm{~nm}$ with a Genesys 6 spectrophotometer (Thermo). Biomass was collected in midexponential growth phase $\left(\mathrm{OD}_{600 \mathrm{~nm}} \approx 2\right)$ by centrifugation $(10$ min, $10000 \mathrm{~g}$, room temperature) and incubated in $5 \mathrm{~mL}$ of $6 \mathrm{~N}$ $\mathrm{HCl}$ at $100{ }^{\circ} \mathrm{C}$ for $15 \mathrm{~h}$. The resulting hydrolysate was dried by evaporation (Rotavapor R-II-HB, Buchi), resuspended in $200 \mu \mathrm{L}$ of $\mathrm{H}_{2} \mathrm{O}$, and cell debris were removed by centrifugation (10 min, $10000 \mathrm{~g}$, room temperature). Sample was then diluted 1600 times before injection, corresponding to $9.38 \mu \mathrm{g}$ of biomass.

Cultivation of rat fibroblast. Mammalian Rat-1 SFv2e-PERK cells were maintained in DMEM high glucose $(25 \mathrm{mM})$ medium supplemented with $4 \mathrm{mM}$ Glutamax (10566016, ThermoFisher), $10 \%$ fatal calf serum (FCS) and $1 \%$ penicillin/streptomycin (P/S) (Gibco). 24 hours before starting the experiment, cells were seeded on cover glasses (ECW 631-1585, VWR). Cells were then washed twice with PBS, incubated with fresh medium containing AP21087 (2 nM, Clontech) to activate the PERK kinase. According to the different labelling time points, the culture medium was replaced with fresh DMEM medium without glucose complemented with $\mathrm{U}-{ }^{13} \mathrm{C}$-glucose $(25 \mathrm{mM}$, Eurisotop) and AP21087 (2 nM). Cells were harvested 24 hours after AP addition with $7 \mathrm{~mL}$ of a quenching solution containing acetonitrile, methanol and water with $0.1 \%$ formic acid at a ratio of $2: 2: 1$ (vol/vol). $375 \mu \mathrm{L}$ of ${ }^{13} \mathrm{C}^{15} \mathrm{~N}$-labelled internal standard were added to each sample, which were then dried in a Speedvac Savant SC250Exp (Thermo Fisher Scientific, Waltham, MA, USA) and resuspended in $750 \mu \mathrm{L}$ of water. Debris were removed by centrifugation at $10000 \mathrm{~g}$ for $5 \mathrm{~min}$, and samples were stored at $80^{\circ} \mathrm{C}$ before LC-HRMS analysis.

LC-HRMS analysis. Amino acids were analyzed by liquid chromatography (Ultimate 3000 HPLC system (Dionex, CA, USA) coupled to a LTQ Orbitrap Velos mass spectrometer (Thermo Fisher Scientific, Waltham, MA, USA) an equipped with a heated electrospray ionization probe, as detailed previously ${ }^{21}$. MS analyses were performed in the positive FTMS mode at a resolution of 60000 (at $\mathrm{m} / \mathrm{z}$ 400) with the following source parameters: capillary temperature was $275{ }^{\circ} \mathrm{C}$, source heater temperature was $250{ }^{\circ} \mathrm{C}$, sheath gas flow rate was 45 , auxiliary gas flow rate was 20, S-Lens RF level was $40 \%$ and source voltage was $5 \mathrm{kV}$. The volume of injection was $20 \mu \mathrm{L}$. Signals of all ${ }^{13} \mathrm{C}$-isotopologues and ${ }^{15} \mathrm{~N}$-isotopologues were extracted with Tracefinder ${ }^{\circledR}$ software (v4.0) with a mass range of $5 \mathrm{ppm}$ (Supplementary Table S6). Data were processed as detailed in the Results section.

Statistical analyses, accuracy and precision. For isotopic measurements, the accuracy was assessed from the closeness of experimental and predicted isotopologue distributions (PT sample) and was formally defined as the mean of absolute differences between measured and predicted values for each isotopologue. For metabolite quantification, the accuracy was determined as the mean of relative difference of concentration determined between the conventional $\left({ }^{13} \mathrm{C}\right.$-IS $)$ and the new approach $\left({ }^{13} \mathrm{C}^{15} \mathrm{~N}\right.$-IS $)$. The precision of all measurements was estimated as the mean value of standard deviations resulting from measurements in triplicate.

\section{RESULTS AND DISCUSSION}

General principle. The proposed approach consists in replacing the classical ${ }^{13} \mathrm{C}$-IS which overlaps the CIDs of metabolites by an IS labelled with other isotope(s) that can be resolved from the ${ }^{13} \mathrm{C}$ isotopologues in the MS spectra (Figure 1). Hence, the signal of IS should not interfere with the ${ }^{13} \mathrm{C}$-mass fractions of interest and thus should not impact the CIDs. This IS still represents a valuable reference for absolute and relative quantification by overcoming the typical drawbacks of LC-MS, such as matrix effects, as well as the partial loss of metabolites that may occur during sampling and sample preparation ${ }^{14}$. Isotopically labelled IS may also correct measurements for ion suppression effects ${ }^{14,22,23}$. Still, special care must be taken to reduce ion suppression when preparing the labelled IS, e.g. by washing the cells during sampling to remove the residual salts ${ }^{14,20,24}$.

In the following sections, we apply and evaluate this approach to investigate amino acids metabolism. These compounds provide a simple but valuable test case since their concentrations and isotopic profiles are of general interest to address a broad range of biological questions in several fields (metabolic engineering, health, systems biology, etc). We considered the possibility of using two distinct IS: i) a doubly labeled ${ }^{13} \mathrm{C}^{15} \mathrm{~N}-\mathrm{IS}$, which can be resolved from the ${ }^{13} \mathrm{C}$-mass fractions of interest at both low and high resolution MS, and ii) a singly-labeled ${ }^{12} \mathrm{C}^{15} \mathrm{~N}-\mathrm{IS}$, which can be resolved only at high and ultra-high resolution MS (typically resolution $>100000)^{25-27}$. For the sake of genericity, we focused here on the ${ }^{13} \mathrm{C}^{15} \mathrm{~N}$-IS since it can be applied with all MS platforms. The internal standard consisted in a hydrolysate of

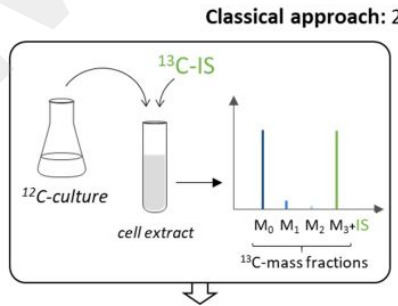

Absolute metabolite concentration

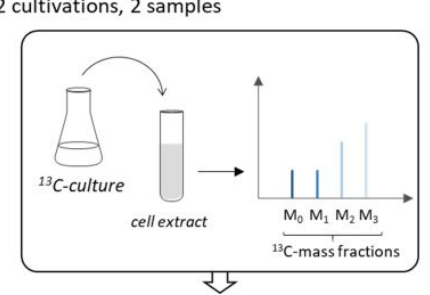

Isotope incorporation (CID)

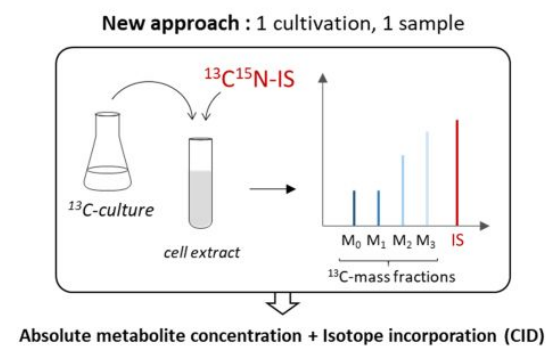

Figure 1. Principle of simultaneous metabolite quantification and isotopic profiling. The classical approach for metabolite quantification and ${ }^{13} \mathrm{C}$-isotopologue profiling requires two independent (unlabeled and labeled) experiments because the ${ }^{13} \mathrm{C}$-IS used for metabolite quantification (in green) cannot be resolved from the ${ }^{13} \mathrm{C}$-mass fraction of interest $\left(\mathrm{M}_{0}-\mathrm{M}_{3}\right.$ for a compound containing three carbon atoms, in blue) in the MS spectra. The proposed approach consists in using another isotopically labeled IS which can be separated from the ${ }^{13} \mathrm{C}$-mass fractions of interest. For instance, a ${ }^{13} \mathrm{C}^{15} \mathrm{~N}$-IS (in red) can be used to analyze amino metabolites. Both information can therefore be collected simultaneously from a unique experiment. 


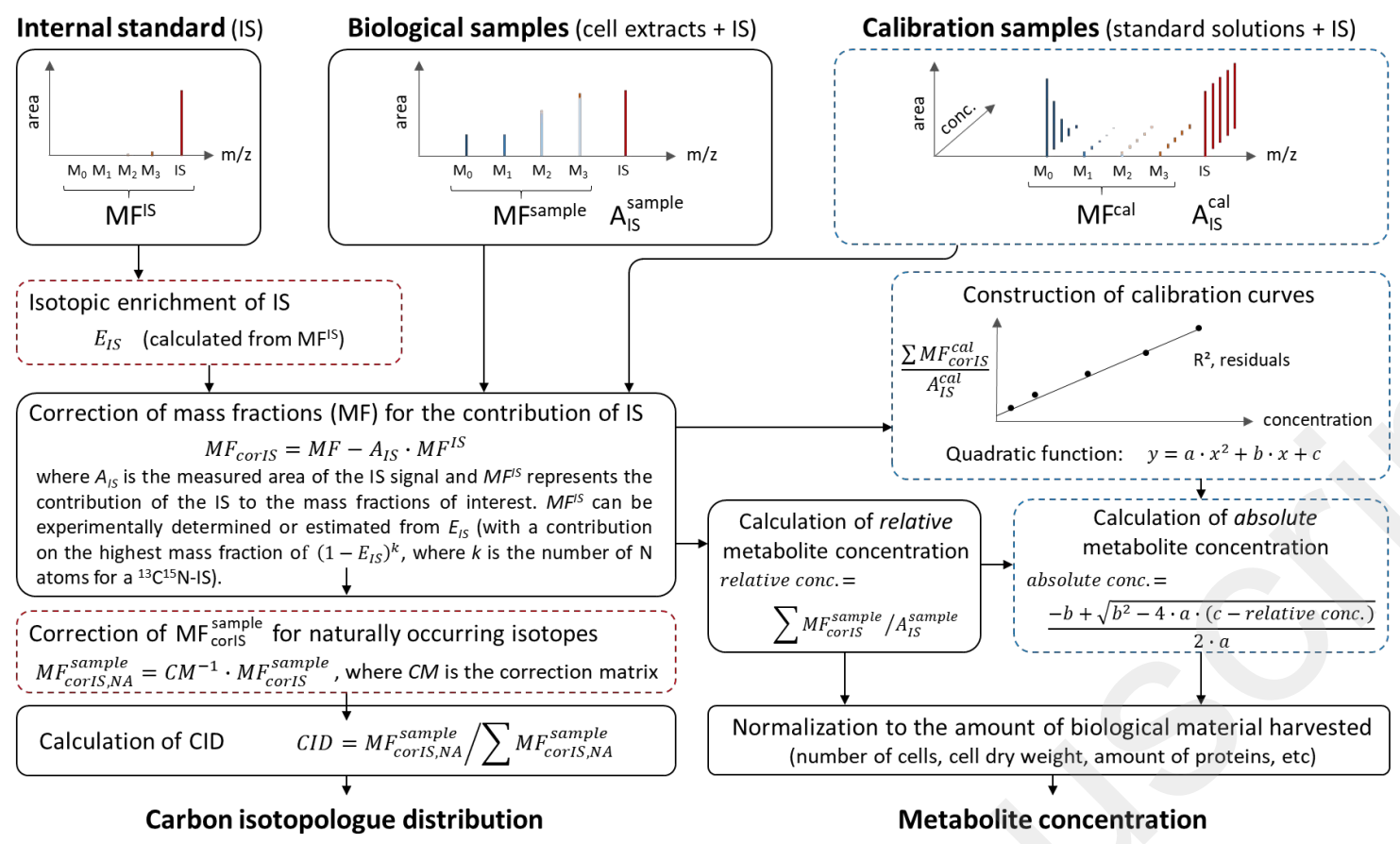

Figure 2. Data processing workflow. Algorithm implemented in IsoQ to calculate the concentration and CID of metabolites. The isotopic enrichment of the internal standard ( $E_{I S}$, calculated from its experimental mass fractions $\left.M F_{I S}\right)$ is used to remove its contribution from the experimental mass fractions measured in biological $\left(M F^{\text {sample }}\right)$ and calibration $\left(M F^{c a l}\right)$ samples. This correction step guarantees the quality of isotopic measurements even for imperfect isotopic purity of the IS. The CID is calculated after correction of $M F_{\text {corl }}$ for the presence of naturally occurring isotopes, and the (relative) concentration is calculated from the ratio between the total signal arising from the metabolite and the IS. Calibration curves constructed with IS added in equal amounts to the unlabeled calibration standards are required for absolute metabolite quantification (blue dotted boxes). Red dotted boxes represent calculation steps carried out by IsoCor ${ }^{25}$.

${ }^{13} \mathrm{C}^{15} \mathrm{~N}$-proteinogenic amino acids obtained by growing Escherichia coli K-12 MG1655 in minimal medium containing ${ }^{15} \mathrm{NH}_{4} \mathrm{Cl}$ as sole nitrogen source and $\mathrm{U}-{ }^{13} \mathrm{C}$-glucose as sole carbon source. Analyses were carried out using the LC-MS method detailed in Heuillet et al. ${ }^{21}$.

This approach requires a dedicated processing workflow (Figure 2) to guarantee the accuracy of the measured concentrations and CIDs. For isotopic measurements, the IS must be characterized to determine its potential contribution to the ${ }^{13} \mathrm{C}$-mass fractions of interest due to the presence of isotopic impurities (i.e. a small fraction of ${ }^{12} \mathrm{C}$ and ${ }^{14} \mathrm{~N}$ atoms) at labeled positions. By measuring the ${ }^{15} \mathrm{~N}$ purity in the IS, the contribution of ${ }^{14} \mathrm{~N}$ impurities can be removed from the measured mass fractions $\left(\mathrm{MF}_{\text {corls }}\right)$, which can then be corrected for the presence of naturally occurring isotopes of non-tracer elements $25\left(\mathrm{MF}_{\text {corIS,NA }}\right)$. The CID is obtained by normalizing the final mass fractions $\left(\mathrm{MF}_{\text {corIS,NA }}\right)$ to 1 . For metabolite quantification, relative metabolite concentrations in biological samples are calculated from the ratio between the total signal arising from the metabolite - i.e. the sum of its ${ }^{13} \mathrm{C}$-mass fractions $\mathrm{MF}_{\text {corls }}$ - and the IS. Absolute metabolite concentrations can be obtained from calibration curves constructed using standard solutions of (unlabeled) metabolites at different concentrations mixed with equal amounts of IS, as detailed in $\mathrm{Wu}$ et al. ${ }^{14}$. This processing workflow was implemented as a Python library, IsoQ, to streamline data processing into an end-to-end environment. IsoQ takes as input data the mass fractions of all metabolites measured in i) the internal standard, ii) the biological samples and iii) the calibration samples, and provides as output their (absolute) concentrations and CIDs (Figure 2). IsoQ and its documentation are available under a GPLv3 open source license at https://github.com/MetaSys-LISBP/IsoQ/.

Validation of isotopic and metabolomics measurements. With our approach, quantification of both metabolite CID and concentration is a complex process based on the measurement of not only one peak but many peaks, to which significant data processing is applied. The utilization of isotopic tracers in ILEs significantly increases the number of peaks (per metabolite) in MS spectra compared to unlabelled samples. This phenomenon is further amplified by the addition of the ${ }^{13} \mathrm{C}^{15} \mathrm{~N}-\mathrm{IS}$, which increases the risk of contamination of peaks of interest by signals arising from other metabolites. Special care must therefore be taken to evaluate the quality of the measurements, which may depend on the metabolite, the analytical method, or the biological matrix (e.g. microorganism, growth condition, sampling and sample preparation procedures). Similarly to other experimental steps (e.g. sampling and sample preparation), this approach should therefore be evaluated in the specific context of each biological study.

We first characterized the ${ }^{13} \mathrm{C}^{15} \mathrm{~N}$-IS by measuring its ${ }^{13} \mathrm{C}$ - and ${ }^{15} \mathrm{~N}$-isotopic purity, which were above $98 \%$ and $99 \%$, respectively, for all amino acids (Supporting Table S1). This high ${ }^{15} \mathrm{~N}$ isotopic enrichment indicates that the ${ }^{13} \mathrm{C}^{15} \mathrm{~N}$-IS should not significantly contribute to the ${ }^{13} \mathrm{C}^{14} \mathrm{~N}$-mass fractions from which the CIDs are quantified. This was evaluated by analyzing a standard isotopic sample (PT sample) with a controlled and predictable distribution of ${ }^{13} \mathrm{C}$-isotopologues which follows the binomial coefficients of Pascal triangle. The nature, production, and qualification of this standard sample have been extensively detailed in a previous report ${ }^{21,28}$. The high correlation between the CIDs quantified in the PT sample with and without addition of the ${ }^{13} \mathrm{C}^{15} \mathrm{~N}$-IS confirmed that the IS does not disturb the measured CIDs $\left(\mathrm{R}^{2}=0.9998\right.$, average difference between both methods of $0.1 \%$, Figure 3A and Supporting Table S2). The accuracy and precision of both approaches were very similar (accuracy $<0.5 \%$ and precision $<0.1 \%)^{21}$. The distribution of differences between 


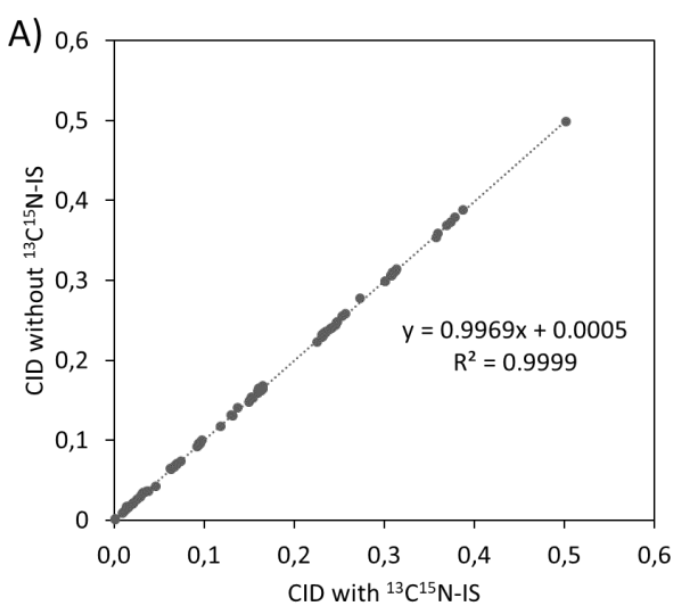

B)

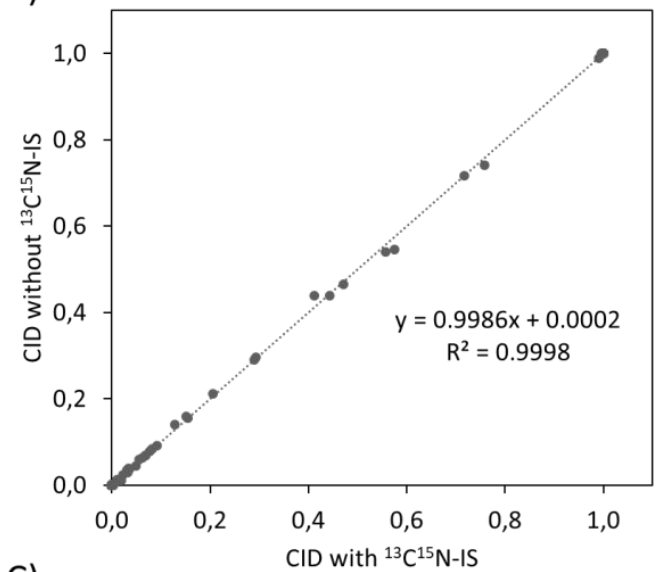

C)

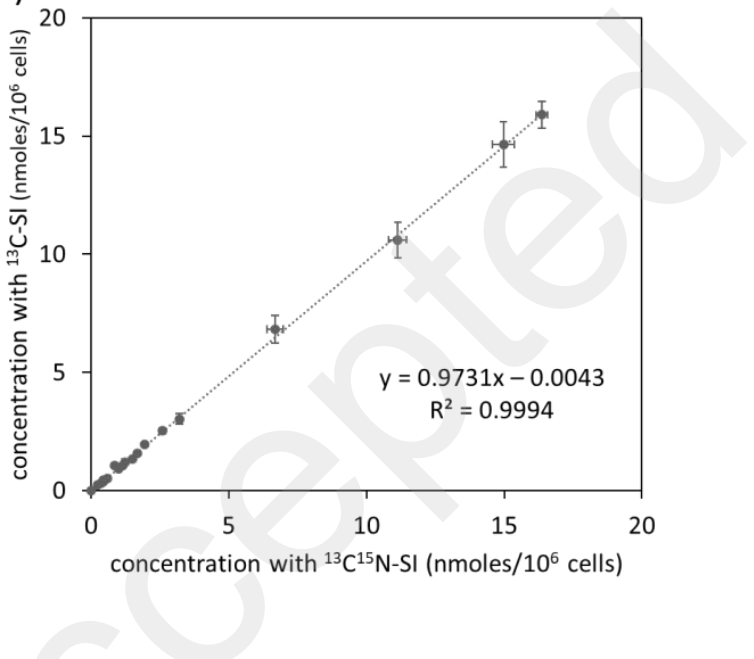

Figure 3. Validation results. The CIDs of amino acids of a reference isotopic sample (PT sample) determined with and without addition of the ${ }^{13} \mathrm{C}^{15} \mathrm{~N}$-IS were in excellent agreement (panel A). This was also verified in rat fibroblasts grown on $\mathrm{U}-{ }^{13} \mathrm{C}$-glucose (panel B). For metabolomics measurements, the concentration of amino acids in rat fibroblast measured with the classical ${ }^{13} \mathrm{C}$-IS and the ${ }^{13} \mathrm{C}^{15} \mathrm{~N}$-IS were also highly consistent (panel C).

predicted and experimental CIDs was centered on zero, which indicates the absence of systematic bias. We also confirmed that the ${ }^{13} \mathrm{C}^{15} \mathrm{~N}$-IS does not disturb the CIDs measured in mammalian cells (rat fibroblast grown on $\mathrm{U}_{-13}^{13} \mathrm{C}$-glucose), which were similar with and without the addition of the ${ }^{13} \mathrm{C}^{15} \mathrm{~N}-\mathrm{IS}\left(\mathrm{R}^{2}=0.9998\right.$, Figure 3B, Supporting Table S3).

We evaluated the approach for absolute metabolite quantification by comparing the intracellular concentration of amino acids in rat fibroblast measured with the classical ${ }^{13} \mathrm{C}$-IS and the novel ${ }^{15} \mathrm{~N}^{13} \mathrm{C}$-IS (Figure 3C, Supporting Table S4). As observed for isotopic analyses, results obtained by both methods showed an excellent agreement $\left(\mathrm{R}^{2}=0.9994\right.$, mean difference of $7 \%$ ) with a similar precision $(<6 \%)$.

Overall, these data demonstrate that the proposed approach is reliable to measure simultaneously metabolite concentrations and isotope incorporation in a unique sample collected from a single cultivation, with a similar precision and accuracy compared to classical approaches which require two samples collected from two independent cultivations.

Dynamic regulation of amino acids metabolism upon activation of the PERK kinase in rat fibroblasts. The proposed approach was exploited to study amino acid metabolism in Rat-1 Fv2E-PERK cells, a rat fibroblastic cell line engineered to stably express a ligand-inducible PERK-Fv2E chimeric protein composed of the protein kinase R (PRK)-like endoplasmic reticulum kinase (PERK) and the FKBP12 (Fv2E) protein that can dimerize in presence of its ligand AP20187 (AP). The PERK kinase is a key component of the unfolded protein response (UPR) that gets activated during endoplasmic reticulum stress. PERK phosphorylates the eukaryotic initiation factor 2 alpha (Eif2a) subunit of the 43S translation pre-initiation complex on serine 51 in order to restrain global protein synthesis. Eif2a concomitantly favors translation of a specific set of proteins that includes the transcription factor ATF4. ATF4 is a downstream effector of various stress response pathways controlling a complex transcriptional program that includes genes involved in metabolism, cytoprotection, and oxidative stress ${ }^{29}$. As previously described $^{30,31}$, addition of $2 \mathrm{nM}$ AP induces the dimerization and activation of the PERK-Fv2E chimeric protein. Consequently, activation of PERK led to increased phosphorylation of Eif2a, as well as increased ATF4 protein level which induces a global cellular response. However, the response of amino acid metabolism to the PERK kinase activation remains largely unknown.

We performed a ${ }^{13} \mathrm{C}$-ILE by growing rat fibroblasts on ${ }^{12} \mathrm{C}$ glucose in the presence or absence of AP. After $18 \mathrm{~h}$ of treatment, cells were switched from ${ }^{12} \mathrm{C}$ - to $\mathrm{U}-{ }^{13} \mathrm{C}$ glucose, and quantified the evolution over time of metabolite pools and of their isotopic profiles (Figure 4 and Supporting Table S5). The concentrations of five amino acids were significantly reduced in response to AP (glutamate, aspartate, alanine, serine and threonine; $\mathrm{p}<0.05$ ). Label was incorporated into five amino acids (proline, glutamate, aspartate, alanine, glutamine) as well as in reduced and oxidized glutathione, indicating that glucose contributes to their biosynthesis. These five compounds correspond to non-essential amino acids which can be synthesized by cellular metabolism ${ }^{32}$. For these amino acids, isotope incorporation was significantly reduced upon activation of the PERK kinase. No labeling was observed in the other amino acids, indicating that they are produced from other carbon sources (e.g. glutamine) and/or directly taken up from the extracellular medium.

Integration of metabolomics and isotopic datasets may provide quantitative information on the biosynthetic flux of amino acids. The labeling dynamics of a given metabolite reflects its turnover rate, which is roughly equivalent to the ratio of the metabolite pool size and the flux through that metabolite pool. The significant decrease of pools observed in response to activation of the PERK kinase is expected to accelerate the transient ${ }^{13} \mathrm{C}$ incorporation ${ }^{6}$. However, the ${ }^{13} \mathrm{C}$-enrichment profiles were slow down in response to AP. This concomitant decrease of both amino 

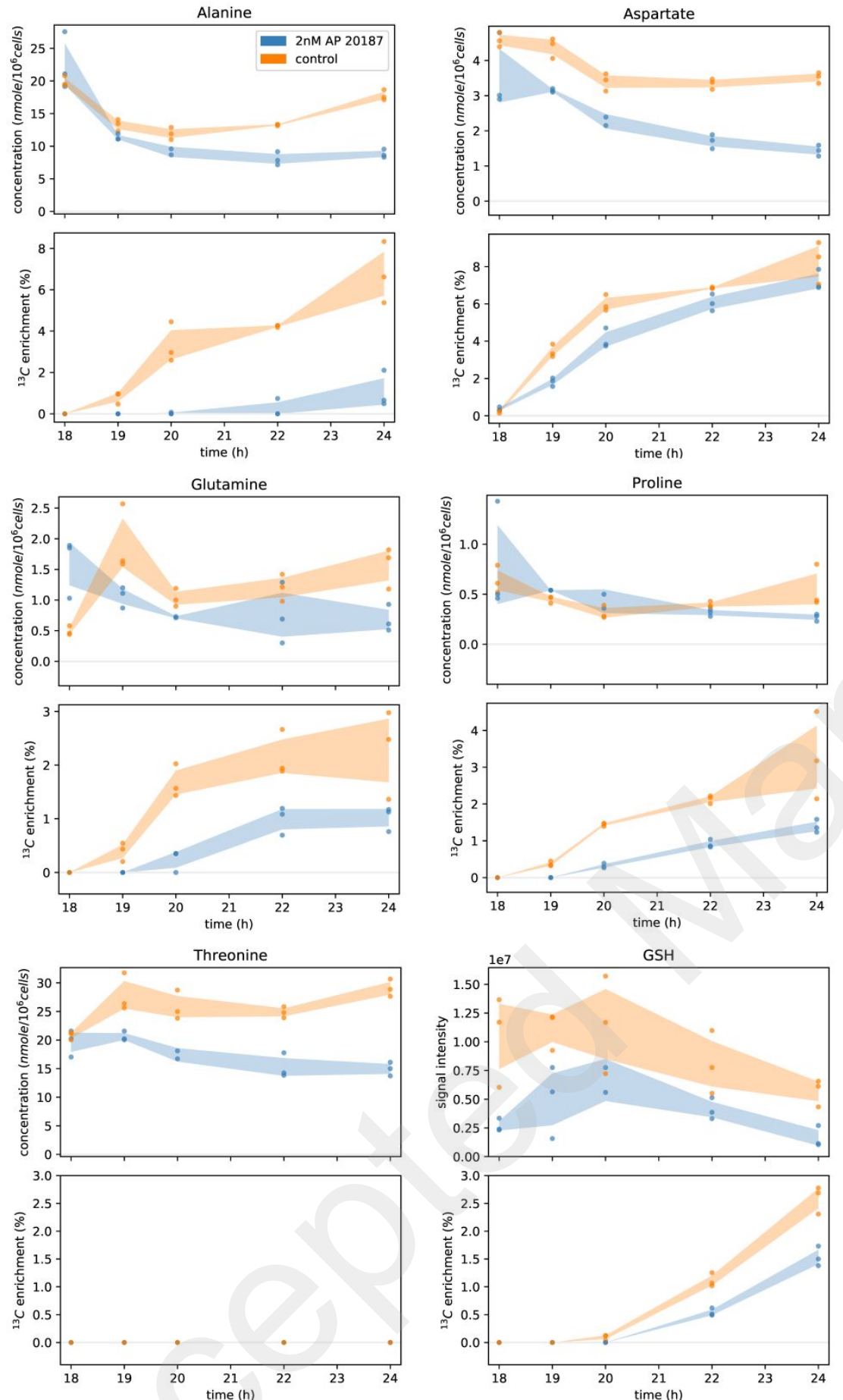
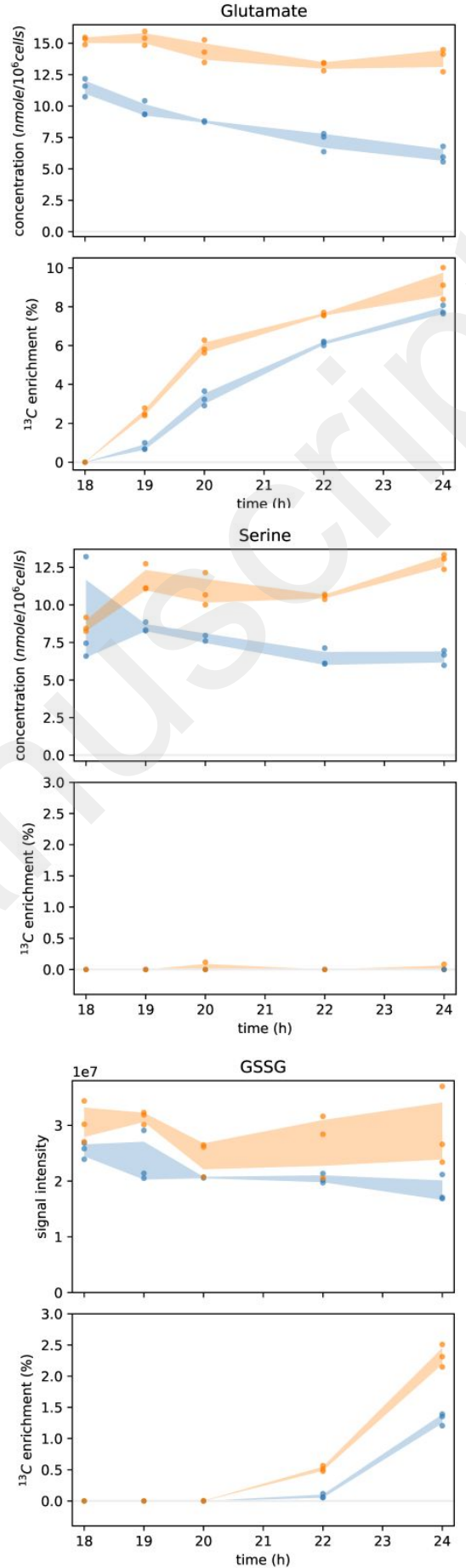

Figure 4. Dynamics of concentration and ${ }^{13} \mathrm{C}$-incorporation into amino acids upon PERK kinase activation in rat fibroblasts. Rat fibroblast were grown on ${ }^{12} \mathrm{C}$-glucose in the presence (blue) or absence (orange) of $\mathrm{AP} 20187$. After $18 \mathrm{~h}$ of treatment, cells were switched from ${ }^{12} \mathrm{C}$ - to U${ }^{13} \mathrm{C}$-glucose, and the concentration and isotope incorporation was monitored into amino acids. Shaded area represents the standard deviation estimated from triplicate measurements (dots). Other amino acids which did not incorporate ${ }^{13} \mathrm{C}$-tracer and with similar concentrations with and without induction of the PERK kinase $(\mathrm{p}>0.05)$ are provided in Supporting Table S5.

acids concentration and isotope dynamics therefore indicates a major reprogramming of amino acids metabolism, which reduces their de novo production from glucose. This reduction of their biosynthetic flux is consistent with the reduced requirements of amino acids for protein synthesis upon action of the PERK kinase. Inferring quantitative flux values from these data would require more sophisticated mathematical models of metabolism, such as dynamic ${ }^{13} \mathrm{C}$-flux models. Still, these results already demonstrate the applicability of the proposed approach to infer flux information on amino acids metabolism in mammalian cells. 


\section{CONCLUSION}

We present a novel approach to measure simultaneously the (absolute or relative) concentrations and isotopologue distributions of metabolites in a unique sample collected from a single cultivation. The basic principle consists in using an isotopically labeled IS which can be resolved from the mass fractions of interest in the MS spectra. Results demonstrate that our approach provides a similar precision and accuracy compared to classical approaches, which require two samples collected from two independent cultivations. Though demonstrated for LCHRMS analysis of amino acids metabolism in rat fibroblast, this approach is generic with respect to the organism, the class of metabolites, and the analytical platform (e.g. low resolution MS). A ${ }^{15} \mathrm{~N}^{13} \mathrm{C}$-IS is sufficient to analyze a broad range of molecules (e.g. $56 \%$ of $E$. coli metabolome contains at least one $\mathrm{N}$ atom). Since the approach and processing workflow developed in this work remain valid for any isotopic tracer and metabolite, other isotopes (e.g. ${ }^{2} \mathrm{H},{ }^{18} \mathrm{O}$ or ${ }^{34} \mathrm{~S}$ ) can be used to analyze specific classes of metabolites.

Combined with recent modeling advances ${ }^{6,7,33}$, this quantitative approach represents a valuable tool for the analysis of metabolic fluxes. Besides significantly reducing experimental and analytical efforts, it may also enhance our capacity to address novel biological questions (e.g. to better understand the mechanistic basis of metabolic variability by integrating self-consistent datasets collected from a single cultivation).

\section{ASSOCIATED CONTENT}

\section{Supporting Information}

Additional information as indicated in the text. This material is available free of charge via the Internet at http://pubs.acs.org/.

\section{AUTHOR INFORMATION}

\section{Corresponding Author}

* Telephone: +33(0)5-61-55-94-07. E-mail: bellvert@insatoulouse.fr.

\section{Author Contributions}

\$These authors contributed equally.

Notes

The authors declare no competing financial interest.

\section{ACKNOWLEDGMENT}

MetaToul (Toulouse metabolomics \& fluxomics facilities, www.metatoul.fr) is part of the French National Infrastructure for Metabolomics and Fluxomics MetaboHUB-ANR-11-INBS-0010 (www.metabohub.fr), and is supported by the Région MidiPyrénées, the ERDF, the SICOVAL and the French Minister of Education \& Research, who are gratefully acknowledged. We thank Baudoin Delépine (MetaSys team, Toulouse Biotechnology Institute, Toulouse, France) for insightful discussions on data processing and visualization. Jean-Charles Portais is grateful to the INSERM for funding a temporary full-time researcher position.

\section{REFERENCES}

(1) Toya, Y.; Shimizu, H. Flux Analysis and Metabolomics for Systematic Metabolic Engineering of Microorganisms. Bioenergy Biorefinery Biomass Innov. Technol. Dev. 2013, 31 (6), 818-826. https://doi.org/10.1016/j.biotechadv.2013.05.002.

(2) Keibler, M. A.; Fendt, S.-M.; Stephanopoulos, G. Expanding the Concepts and Tools of Metabolic
Engineering to Elucidate Cancer Metabolism. Biotechnol.

Prog. 2012, 28 (6), 1409-1418.

https://doi.org/10.1002/btpr.1629.

(3) Sévin, D. C.; Kuehne, A.; Zamboni, N.; Sauer, U. Biological Insights through Nontargeted Metabolomics. Syst. Biol. • Nanobiotechnology 2015, 34, 1-8. https://doi.org/10.1016/j.copbio.2014.10.001.

Heux, S.; Bergès, C.; Millard, P.; Portais, J.-C.; Létisse, F. Recent Advances in High-Throughput 13C-Fluxomics. Anal. Biotechnol. 2017, 43, 104-109.

https://doi.org/10.1016/j.copbio.2016.10.010. Christodoulou, D.; Kuehne, A.; Estermann, A.; Fuhrer, T.; Lang, P.; Sauer, U. Reserve Flux Capacity in the Pentose Phosphate Pathway by NADPH Binding Is Conserved across Kingdoms. iScience 2019, 19, 11331144. https://doi.org/10.1016/j.isci.2019.08.047.

Buescher, J. M.; Antoniewicz, M. R.; Boros, L. G.; Burgess, S. C.; Brunengraber, H.; Clish, C. B.; DeBerardinis, R. J.; Feron, O.; Frezza, C.; Ghesquiere, B.; Gottlieb, E.; Hiller, K.; Jones, R. G.; Kamphorst, J. J.; Kibbey, R. G.; Kimmelman, A. C.; Locasale, J. W.; Lunt, S. Y.; Maddocks, O. D. K.; Malloy, C.; Metallo, C. M.; Meuillet, E. J.; Munger, J.; Nöh, K.; Rabinowitz, J. D.; Ralser, M.; Sauer, U.; Stephanopoulos, G.; St-Pierre, J.; Tennant, D. A.; Wittmann, C.; Vander Heiden, M. G.; Vazquez, A.; Vousden, K.; Young, J. D.; Zamboni, N.; Fendt, S.-M. A Roadmap for Interpreting (13)C Metabolite Labeling Patterns from Cells. Curr. Opin. Biotechnol. 2015, 34, 189-201.

https://doi.org/10.1016/j.copbio.2015.02.003.

Millard, P.; Schmitt, U.; Kiefer, P.; Vorholt, J. A.; Heux, S.; Portais, J.-C. ScalaFlux: A Scalable Approach to Quantify Fluxes in Metabolic Subnetworks. bioRxiv 2019, 735308. https://doi.org/10.1101/735308.

Delépine, B.; López, M. G.; Carnicer, M.; Vicente, C. M.; Wendisch, V. F.; Heux, S. Charting the Metabolic Landscape of the Facultative Methylotroph $<$ em $>$ Bacillus Methanolicus $</$ Em $>$. bioRxiv 2019, 858514.

https://doi.org/10.1101/858514.

(9) Xu, Y.-F.; Amador-Noguez, D.; Reaves, M. L.; Feng, X.J.; Rabinowitz, J. D. Ultrasensitive Regulation of Anapleurosis via Allosteric Activation of PEP Carboxylase. Nat. Chem. Biol. 2012, 8 (6), 562-568. https://doi.org/10.1038/nchembio.941.

(10) Enjalbert, B.; Millard, P.; Dinclaux, M.; Portais, J.-C.; Létisse, F. Acetate Fluxes in Escherichia Coli Are Determined by the Thermodynamic Control of the PtaAckA Pathway. Sci. Rep. 2017, 7, 42135-42135. https://doi.org/10.1038/srep42135.

Millard, P.; Portais, J.-C.; Mendes, P. Impact of Kinetic Isotope Effects in Isotopic Studies of Metabolic Systems. BMC Syst. Biol. 2015, 9 (1), 64. https://doi.org/10.1186/s12918-015-0213-8. Millard, P.; Smallbone, K.; Mendes, P. Metabolic Regulation Is Sufficient for Global and Robust Coordination of Glucose Uptake, Catabolism, Energy Production and Growth in Escherichia Coli. PLoS 
Comput. Biol. 2017, 13 (2), e1005396-e1005396.

https://doi.org/10.1371/journal.pcbi.1005396.

(13) Bennett, B. D.; Yuan, J.; Kimball, E. H.; Rabinowitz, J.

D. Absolute Quantitation of Intracellular Metabolite

Concentrations by an Isotope Ratio-Based Approach.

Nat. Protoc. 2008, 3 (8), 1299-1311.

https://doi.org/10.1038/nprot.2008.107.

(14) Wu, L.; Mashego, M. R.; van Dam, J. C.; Proell, A. M.; Vinke, J. L.; Ras, C.; van Winden, W. A.; van Gulik, W. M.; Heijnen, J. J. Quantitative Analysis of the Microbial Metabolome by Isotope Dilution Mass Spectrometry Using Uniformly 13C-Labeled Cell Extracts as Internal Standards. Anal. Biochem. 2005, 336 (2), 164-171. https://doi.org/10.1016/j.ab.2004.09.001.

(15) You, L.; Zhang, B.; Tang, Y. J. Application of Stable Isotope-Assisted Metabolomics for Cell Metabolism Studies. Metabolites 2014, 4 (2), 142-165. https://doi.org/10.3390/metabo4020142.

(16) Lu, W.; Su, X.; Klein, M. S.; Lewis, I. A.; Fiehn, O.; Rabinowitz, J. D. Metabolite Measurement: Pitfalls to Avoid and Practices to Follow. Annu. Rev. Biochem. 2017, 86 (1), 277-304. https://doi.org/10.1146/annurevbiochem-061516-044952.

(17) Weindl, D.; Wegner, A.; Jäger, C.; Hiller, K. Isotopologue Ratio Normalization for Non-Targeted Metabolomics. J. Chromatogr. A 2015, 1389, 112-119. https://doi.org/10.1016/j.chroma.2015.02.025.

(18) Patacq, C.; Chaudet, N.; Létisse, F. Absolute Quantification of PpGpp and PppGpp by Double-Spike Isotope Dilution Ion Chromatography-High-Resolution Mass Spectrometry. Anal. Chem. 2018, 90 (18), 1071510723. https://doi.org/10.1021/acs.analchem.8b00829.

(19) Hegeman, A. D.; Schulte, C. F.; Cui, Q.; Lewis, I. A.; Huttlin, E. L.; Eghbalnia, H.; Harms, A. C.; Ulrich, E. L.; Markley, J. L.; Sussman, M. R. Stable Isotope Assisted Assignment of Elemental Compositions for Metabolomics. Anal. Chem. 2007, 79 (18), 6912-6921. https://doi.org/10.1021/ac070346t.

(20) Millard, P.; Massou, S.; Wittmann, C.; Portais, J.-C.; Létisse, F. Sampling of Intracellular Metabolites for Stationary and Non-Stationary (13)C Metabolic Flux Analysis in Escherichia Coli. Anal. Biochem. 2014, 465, 38-49. https://doi.org/10.1016/j.ab.2014.07.026.

(21) Heuillet, M.; Bellvert, F.; Cahoreau, E.; Letisse, F.; Millard, P.; Portais, J.-C. Methodology for the Validation of Isotopic Analyses by Mass Spectrometry in StableIsotope Labeling Experiments. Anal. Chem. 2018, 90 (3), 1852-1860. https://doi.org/10.1021/acs.analchem.7b03886.

(22) Mashego, M. R.; Wu, L.; Van Dam, J. C.; Ras, C.; Vinke, J. L.; Van Winden, W. A.; Van Gulik, W. M.; Heijnen, J. J. MIRACLE: Mass Isotopomer Ratio Analysis of U13C-Labeled Extracts. A New Method for Accurate Quantification of Changes in Concentrations of Intracellular Metabolites. Biotechnol. Bioeng. 2004, 85 (6), 620-628. https://doi.org/10.1002/bit.10907.
Sojo, L. E.; Lum, G.; Chee, P. Internal Standard Signal Suppression by Co-Eluting Analyte in Isotope Dilution LC-ESI-MS. Analyst 2003, 128 (1), 51-54. https://doi.org/10.1039/B209521C.

(24) Kiefer, P.; Portais, J.-C.; Vorholt, J. A. Quantitative Metabolome Analysis Using Liquid ChromatographyHigh-Resolution Mass Spectrometry. Anal. Biochem. 2008, 382 (2), 94-100. https://doi.org/10.1016/j.ab.2008.07.010.

(25) Millard, P.; Delépine, B.; Guionnet, M.; Heuillet, M.; Bellvert, F.; Létisse, F. IsoCor: Isotope Correction for High-Resolution MS Labeling Experiments.

Bioinformatics 2019, 35 (21), 4484-4487. https://doi.org/10.1093/bioinformatics/btz209.

(26) Khodjaniyazova, S.; Nazari, M.; Garrard, K. P.; Matos, M. P. V.; Jackson, G. P.; Muddiman, D. C. Characterization of the Spectral Accuracy of an Orbitrap Mass Analyzer Using Isotope Ratio Mass Spectrometry. Anal. Chem. 2018, 90 (3), 1897-1906. https://doi.org/10.1021/acs.analchem.7b03983.

(27) Su, X.; Lu, W.; Rabinowitz, J. D. Metabolite Spectral Accuracy on Orbitraps. Anal. Chem. 2017, 89 (11), 5940-5948. https://doi.org/10.1021/acs.analchem.7b00396. Millard, P.; Massou, S.; Portais, J.-C.; Létisse, F. Isotopic Studies of Metabolic Systems by Mass Spectrometry: Using Pascal's Triangle to Produce Biological Standards with Fully Controlled Labeling Patterns. Anal. Chem. 2014, 86 (20), 10288-10295. https://doi.org/10.1021/ac502490g.

(29) Harding, H. P.; Zhang, Y.; Zeng, H.; Novoa, I.; Lu, P. D.; Calfon, M.; Sadri, N.; Yun, C.; Popko, B.; Paules, R.; Stojdl, D. F.; Bell, J. C.; Hettmann, T.; Leiden, J. M.; Ron, D. An Integrated Stress Response Regulates Amino Acid Metabolism and Resistance to Oxidative Stress. Mol. Cell 2003, 11 (3), 619-633. https://doi.org/10.1016/S1097-2765(03)00105-9. Miyake, M.; Kuroda, M.; Kiyonari, H.; Takehana, K.; Hisanaga, S.; Morimoto, M.; Zhang, J.; Oyadomari, M.; Sakaue, H.; Oyadomari, S. Ligand-Induced Rapid Skeletal Muscle Atrophy in HSA-Fv2E-PERK Transgenic Mice. PloS One 2017, 12 (6), e0179955e0179955. https://doi.org/10.1371/journal.pone.0179955. (31) Huber, A.-L.; Lebeau, J.; Guillaumot, P.; Pétrilli, V.; Malek, M.; Chilloux, J.; Fauvet, F.; Payen, L.; Kfoury, A.; Renno, T.; Chevet, E.; Manié, S. N. P58IPKMediated Attenuation of the Proapoptotic PERK-CHOP Pathway Allows Malignant Progression upon Low Glucose. Mol. Cell 2013, 49 (6), 1049-1059. https://doi.org/10.1016/j.molcel.2013.01.009. Eagle, H. The Specific Amino Acid Requirements of Mammalian Cell (Strain L) in Tissue Culture. J. Biol. Chem. 1955, 214, 839-852.

Jang, C.; Chen, L.; Rabinowitz, J. D. Metabolomics and Isotope Tracing. Cell 2018, 173 (4), 822-837. https://doi.org/10.1016/j.cell.2018.03.055. 
For Table of Contents Only

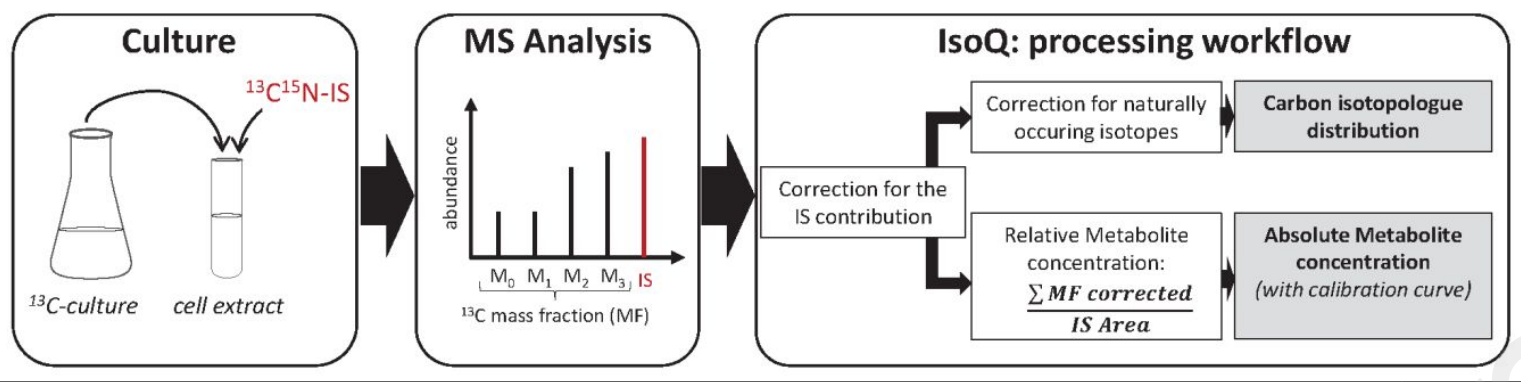


Classical approach: 2 cultivations, 2 samples

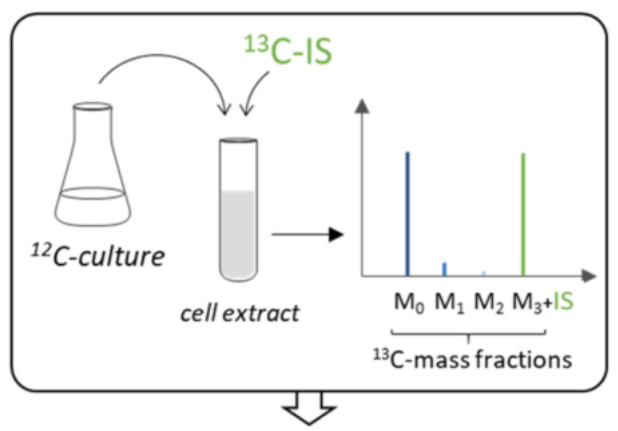

Absolute metabolite concentration

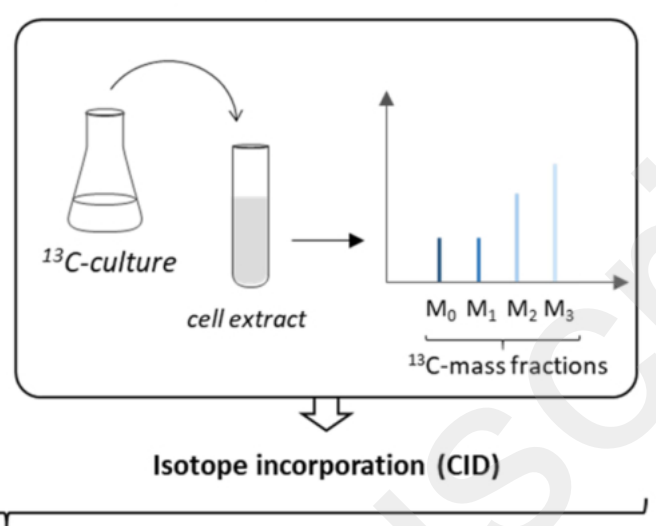

New approach : 1 cultivation, 1 sample

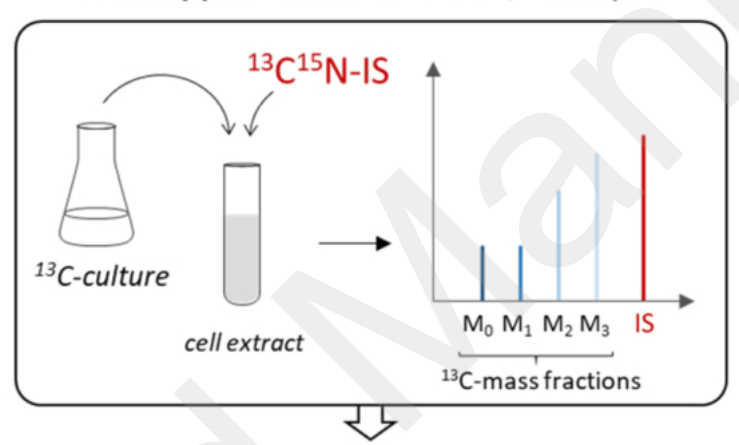

Absolute metabolite concentration + Isotope incorporation (CID)

Figure 1. Principle of simultaneous metabolite quantification and isotopic profiling. The classical approach for metabolite quantification and ${ }^{13} \mathrm{C}$-isotopologue profiling requires two independent (unlabeled and labeled) experiments because the ${ }^{13} \mathrm{C}$-IS used for metabolite quantification (in green) cannot be resolved from the ${ }^{13} \mathrm{C}$-mass fraction of interest $\left(\mathrm{M}_{0}-\mathrm{M}_{3}\right.$ for a compound containing three carbon atoms, in blue) in the MS spectra. The proposed approach consists in using another isotopically labeled IS which can be separated from the ${ }^{13} \mathrm{C}$-mass fractions of interest. For instance, a ${ }^{13} \mathrm{C}^{15} \mathrm{~N}$-IS (in red) can be used to analyze amino metabolites. Both information can therefore be collected simultaneously from a unique experiment.

$82 \times 72 \mathrm{~mm}(300 \times 300 \mathrm{DPI})$ 


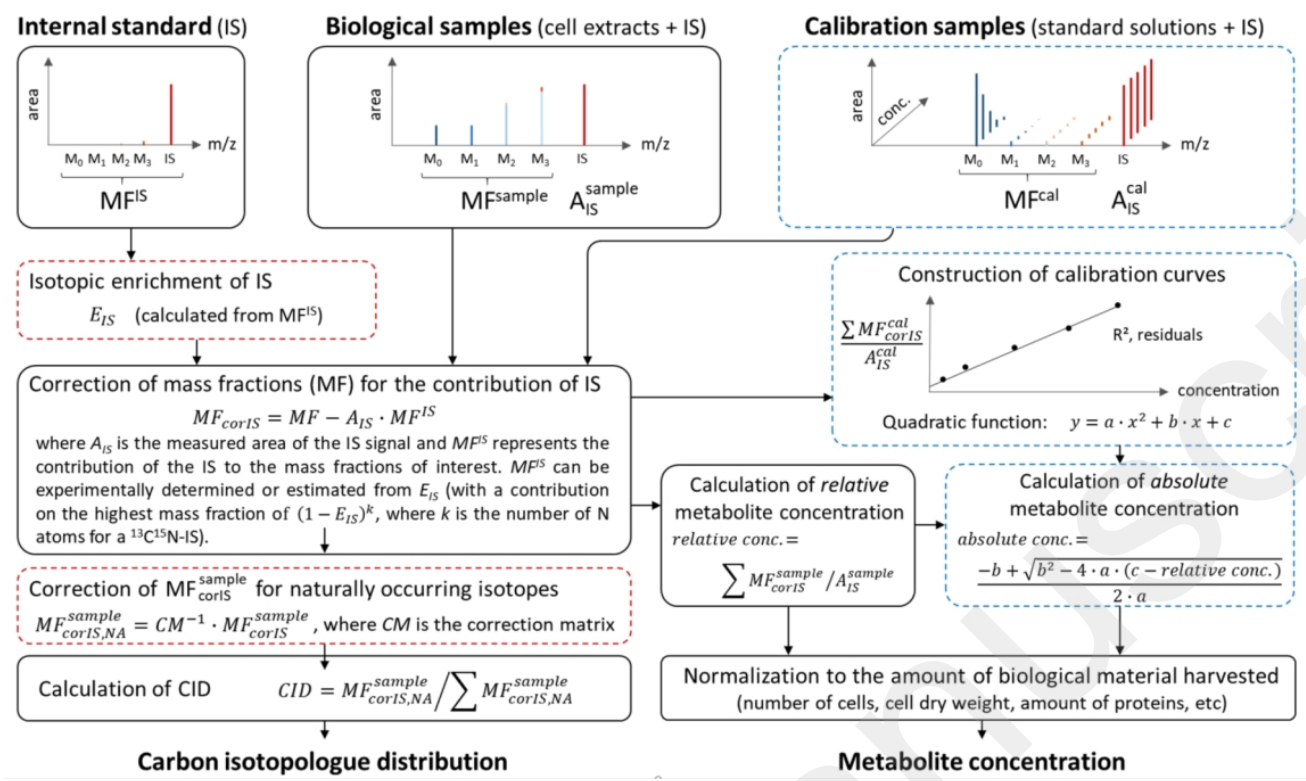

Figure 2. Data processing workflow. Algorithm implemented in IsoQ to calculate the concentration and CID of metabolites. The isotopic enrichment of the internal standard ( $E_{I S}$, calculated from its experimental mass fractions MF $_{I S}$ ) is used to remove its contribution from the experimental mass fractions measured in biological $\left(\mathrm{MF}_{\text {sample}}\right)$ and calibration $\left(\mathrm{MF}_{\mathrm{cal}}\right)$ samples. This correction step guarantees the quality of isotopic measurements even for imperfect isotopic purity of the IS. The CID is calculated after correction of MF $_{\text {corIS }}$ for the presence of naturally occurring isotopes, and the (relative) concentration is calculated from the ratio between the total signal arising from the metabolite and the IS. Calibration curves constructed with IS added in equal amounts to the unlabeled calibration standards are required for absolute metabolite quantification (blue dotted boxes). Red dotted boxes represent calculation steps carried out by IsoCor.

$151 \times 90 \mathrm{~mm}(300 \times 300 \mathrm{DPI})$ 


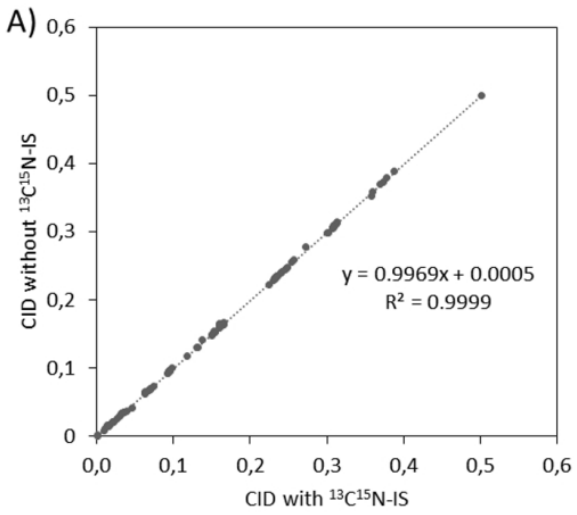

B)
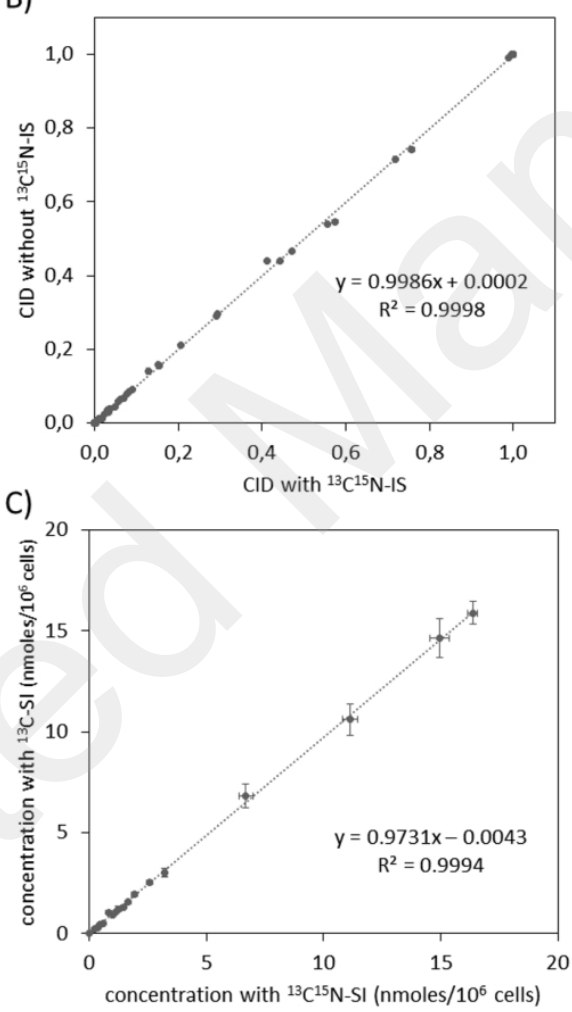

Figure 3. Validation results. The CIDs of amino acids of a reference isotopic sample (PT sample) determined with and without addition of the ${ }^{13} \mathrm{C}^{15} \mathrm{~N}$-IS were in excellent agreement (panel $\mathrm{A}$ ). This was also verified in rat fibroblasts grown on $\mathrm{U}^{13} \mathrm{C}$-glucose (panel B). For metabolomics measurements, the concentration of amino acids in rat fibroblast measured with the classical ${ }^{13} \mathrm{C}$-IS and the ${ }^{13} \mathrm{C}^{15} \mathrm{~N}$-IS were also highly consistent (panel C).

$70 \times 188 \mathrm{~mm}(300 \times 300 \mathrm{DPI})$ 

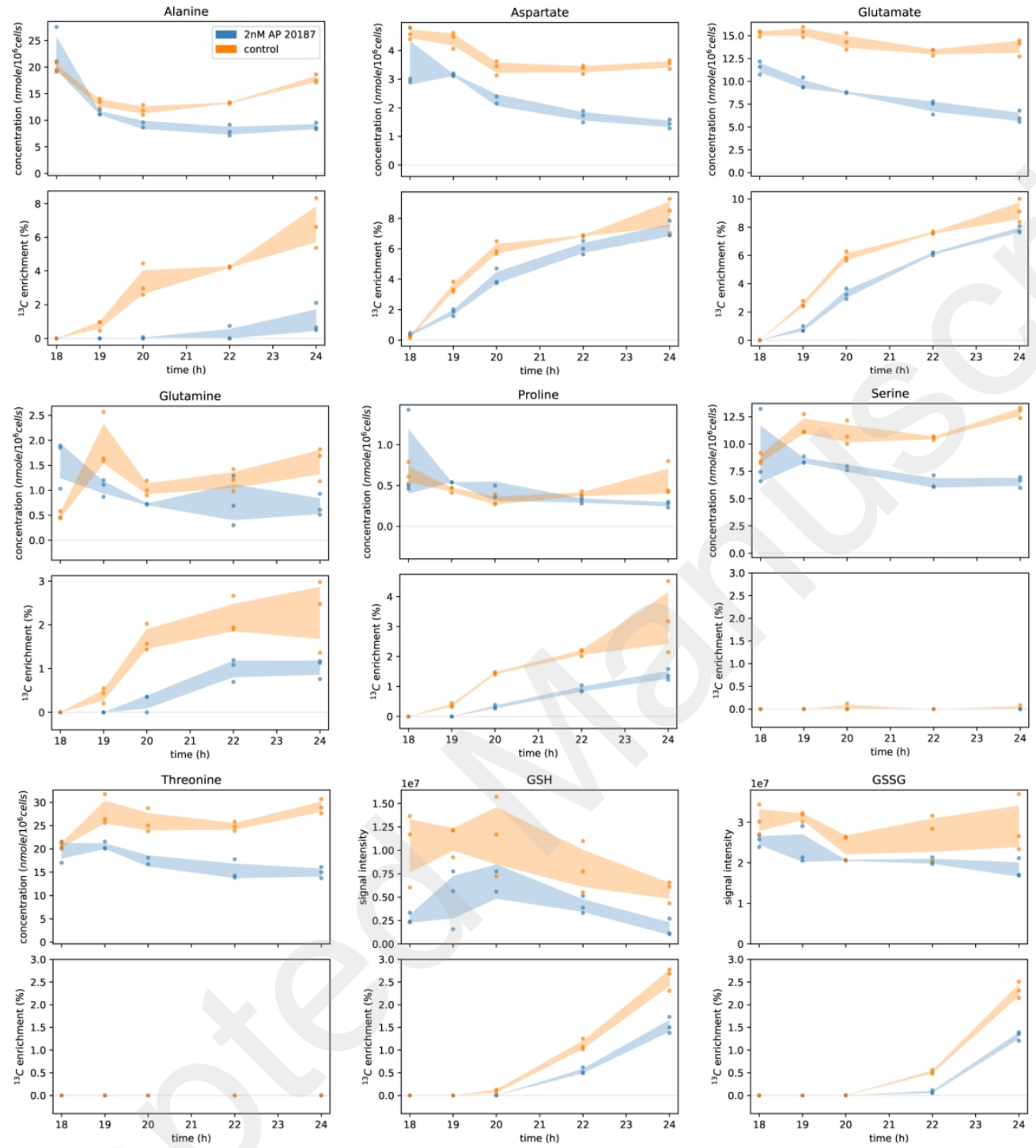

Figure 4. Dynamics of concentration and ${ }^{13} \mathrm{C}$-incorporation into amino acids upon PERK kinase activation in rat fibroblasts. Rat fibroblast were grown on ${ }^{12} \mathrm{C}$-glucose in the presence (blue) or absence (orange) of AP20187. After $18 \mathrm{~h}$ of treatment, cells were switched from ${ }^{12} \mathrm{C}$ - to $\mathrm{U}-{ }^{13} \mathrm{C}$-glucose, and the concentration and isotope incorporation was monitored into amino acids. Shaded area represents the standard deviation estimated from triplicate measurements (dots). Other amino acids which did not incorporate ${ }^{13} \mathrm{C}$-tracer and with similar concentrations with and without induction of the PERK kinase $(p>0.05)$ are provided in Supporting Table S5.

$159 \times 177 \mathrm{~mm}(300 \times 300 \mathrm{DPI})$ 